\title{
Impact of sepsis on risk of postoperative arterial and venous thromboses: large prospective cohort study
}

\author{
@) (1) $\Theta$ OPEN ACCESS
}

\author{
Jacques D Donzé research associate ${ }^{123}$, Paul M Ridker professor ${ }^{345}$, Samuel R G Finlayson \\ professor and chair ${ }^{6}$, David W Bates professor ${ }^{23}$
}

'Division of General Internal Medicine, Bern University Hospital, 3010 Bern, Switzerland; ${ }^{2}$ Division of General Internal Medicine and Primary Care, Brigham and Women's Hospital, 02120 Boston, Massachusetts, USA; ${ }^{3}$ Harvard Medical School, 02115 Boston, Massachusetts, USA; ${ }^{4}$ Center for Cardiovascular Disease Prevention, Division of Preventive Medicine, Brigham and Women's Hospital, 02215 Boston, MA; ${ }^{5}$ Division of Cardiovascular Medicine, Brigham and Women's Hospital, 02215 Boston, MA, USA; ${ }^{6}$ Department of Surgery, University of Utah, 84132 Salt Lake City, UT, USA

\begin{abstract}
Objectives To evaluate the impact of preoperative sepsis on risk of postoperative arterial and venous thromboses.

Design Prospective cohort study using the National Surgical Quality Improvement Program database of the American College of Surgeons (ACS-NSQIP).
\end{abstract}

Setting Inpatient and outpatient procedures in 374 hospitals of all types across the United States, 2005-12.

Participants 2305380 adults who underwent surgical procedures.

Main outcome measures Arterial thrombosis (myocardial infarction or stroke) and venous thrombosis (deep venous thrombosis or pulmonary embolism) in the 30 days after surgery.

Results Among all surgical procedures, patients with preoperative systemic inflammatory response syndrome or any sepsis had three times the odds of having an arterial or venous postoperative thrombosis (odds ratio $3.1,95 \%$ confidence interval 3.0 to 3.1$)$. The adjusted odds ratios were 2.7 (2.5 to 2.8 ) for arterial thrombosis and 3.3 (3.2 to 3.4 ) for venous thrombosis. The adjusted odds ratios for thrombosis were 2.5 (2.4 to 2.6) in patients with systemic inflammatory response syndrome, 3.3 (3.1 to 3.4 ) in patients with sepsis, and 5.7 (5.4 to 6.1) in patients with severe sepsis, compared with patients without any systemic inflammation. In patients with preoperative sepsis, both emergency and elective surgical procedures had a twofold increased odds of thrombosis.

Conclusions Preoperative sepsis represents an important independent risk factor for both arterial and venous thromboses. The risk of thrombosis increases with the severity of the inflammatory response and is higher in both emergent and elective surgical procedures. Suspicion of thrombosis should be higher in patients with sepsis who undergo surgery.

\section{Introduction}

Arterial and venous thromboses are common serious postoperative complications. More than $4 \%$ of surgical procedures are followed by an arterial thrombosis ${ }^{1}$ and at least another $4 \%$ by venous thrombosis. ${ }^{23}$ Several risk factors have been identified, such as existing coronary atherosclerosis for the former and older age, male sex, previous venous thromboembolism, and malignancy for the latter. There are, however, few data on the risk of postoperative thrombosis associated with infection.

Systemic infections with their consequent inflammation and hypercoagulable state might affect risk of postoperative thrombosis. Indeed, a persistent proinflammatory response has long been recognised as a major determinant of myocardial infarction and stroke as well as deep vein thrombosis and pulmonary embolism. ${ }^{4}$ Similarly, infection can activate a hypercoagulable state, inducing platelet activation, production of tissue factor, and increased fibrin turnover, which can all lead to thrombotic complications. ${ }^{6-10}$ Finally, indirect factors such as hypoxaemia and hypotension can also represent triggers for arterial thrombosis.

Better evaluation of the impact of infection and its severity in postoperative thrombotic complication could help in the prevention of thrombotic events among surgical patients. To determine whether an association exists between preoperative sepsis and the risk of arterial and venous thromboses in the postoperative period, we compared the risk of arterial and venous thromboses in patients with and without preoperative sepsis in a large multicentre surgical cohort.

\section{Methods}

\section{Setting}

We used data from the American College of Surgeons National Surgical Quality Improvement Program (ACS-NSQIP). ${ }^{11}$ The ACS-NSQIP is a validated prospective cohort study of a random 
sample of inpatients and outpatients undergoing non-trauma major surgery in up to 374 academic and community hospital across the United States. Trained abstractors use strict standard definitions to collect data at an institutional level, with regular audit to ensure reliability and quality. Data collected include preoperative risk factors, procedures performed by Current Procedural Terminology (CPT) code, and postoperative complications occurring within 30 days of the index operation. Our study followed strengthening of reporting observational studies in epidemiology (STROBE) guidelines. ${ }^{12}$

\section{Study population}

We evaluated all adult patients aged $\geq 18$ who underwent non-trauma major surgery from 1 July 2005 to 31 December 2012. A surgical procedure was considered as major for all procedures carried out under general, spinal, or epidural anaesthesia, as well as the following procedures regardless of the type of anaesthesia: carotid endarterectomy, inguinal hernia repair, parathyroidectomy, thyroidectomy, breast lumpectomy, endovascular abdominal aortic aneurysm repair. Patients were not eligible if they had had a previous operative procedure in the past 30 days. We abstracted the following elements from the database for analysis: age, sex, race, body mass index (BMI), smoking status, treated arterial hypertension, diagnosis of diabetes, severe renal failure, disseminated cancer, bleeding disorder, surgical subspecialty, emergency versus non-emergency surgical procedure, and local versus general anaesthesia. An emergency procedure is performed within a short interval of time between diagnosis or the onset of related preoperative symptoms. Because non-emergency surgical procedures can include semi-urgent cases, we also used the elective and non-elective criteria available only in the 2011-2012 datasets. Patients undergoing elective procedures are brought to the hospital or facility for a scheduled surgery from their home or normal living situation on the day that the procedure is performed.

\section{Exposure}

Trained abstractors at each site identified and categorised patients with a systemic inflammatory response during the 48 hours before the surgical procedure according to systemic inflammatory response syndrome (SIRS), sepsis, or severe sepsis/septic shock. ${ }^{13}$ The systemic inflammatory response syndrome was defined by the presence of two or more of temperature $>38^{\circ} \mathrm{C}$ or $<36^{\circ} \mathrm{C}$; heart rate $>90$ beats/minute; respiratory rate $>20$ breaths/minute or a $\mathrm{PaCO}_{2}<32 \mathrm{~mm} \mathrm{Hg}$ $(<4.3 \mathrm{kPa})$; white blood cell count $>12000 \mathrm{cell} / \mathrm{mm}^{3},<4000$ cells $/ \mathrm{mm}^{3}$, or $>10 \%$ immature band forms; or anion gap acidosis $(>12 \mathrm{mEq} / \mathrm{L})$. Sepsis was defined as systemic inflammatory response syndrome plus one of a positive result on blood culture, clinical documentation of purulence, or positive result on culture from any site thought to be causative. Severe sepsis/septic shock was defined as the presence of sepsis associated with an organ and/or circulatory dysfunction (such as oliguria, acute alteration in mental status, acute respiratory distress/hypotension, requirement for inotropic or vasopressor agents).

By definition, systemic inflammatory response syndrome does not include inflammatory processes in which a bacterial infection is already proved. Therefore, it includes other inflammation processes, such as latent bacterial infection, viral infection (for example, sore throat, bronchitis, flu), or an inflammatory response without infection.

\section{Study outcome}

Our primary outcomes were any arterial or venous thrombosis that occurred intraoperatively or within 30 days after a surgical procedure, including acute myocardial infarction or stroke for arterial thrombosis and deep venous thrombosis or pulmonary embolism for venous thrombosis. Trained abstractors at each site determined acute myocardial infarction according to the following definition: ST elevation $>1 \mathrm{~mm}$ in two or more contiguous leads on the electrocardiogram, new left bundle branch, new $\mathrm{Q}$ wave in two of more contiguous leads, new increase in troponin concentrations to over three times the upper concentration of the reference range in the setting of suspected myocardial ischaemia, or myocardial infarction diagnosed by a physician. A stroke was an embolic, thrombotic, or

haemorrhagic vascular event or stroke with motor, sensory, or cognitive dysfunction (such as hemiplegia, hemiparesis, aphasia, sensory deficit, impaired memory) that persisted for 24 or more hours. A deep venous thrombosis was defined as a new blood clot or thrombus within the venous system confirmed by duplex ultrasongraphy, venography, or computed tomography, and the patient must have been treated with anticoagulation and/or placement of vena cava filter or clipping of the vena cava. A pulmonary embolism was defined by the presence of a blood clot in a pulmonary artery with subsequent obstruction of blood supply to the lung parenchyma or if the patient had a ventilation-perfusion scan interpreted as high probability of pulmonary embolism or a positive result on spiral computed tomography, transoesophageal echocardiography, pulmonary arteriography, or computed tomography angiography. Secondary outcomes were the acute myocardial infarction, stroke, deep venous thrombosis and pulmonary embolism separately, as well as overall 30 day postoperative mortality.

\section{Statistical analysis}

Proportions for baseline characteristics were calculated for patients without any systemic inflammatory response, patients with any systemic inflammatory response or sepsis, patients with systemic inflammatory response syndrome, patients with sepsis, and patients with severe sepsis/septic shock within 48 hours before the surgical procedure. For the four groups with a preoperative systemic inflammatory response, we calculated crude and adjusted odds ratios for the outcomes. To obtain the adjusted odds ratios with $95 \%$ confidence intervals, we adjusted multivariable logistic regression for age, sex, diabetes mellitus, smoking status, treated arterial hypertension, renal failure, cancer, bleeding disorder or anticoagulation, general anaesthesia, and surgical subspecialty. Missing data were extremely rare (four covariates had missing data for one or more case(s), with a maximum of $0.02 \%$ ), but if present they were coded as dummy variables in the analysis. When no information on exposure (preoperative sepsis) was available, the case was excluded from the analysis. Because missing data for exposure are missing at random (that is, without relation to the presence or absence of the exposure), validity of the results is preserved. ${ }^{14}$

No outcome data were missing. We performed stratified analyses separately for the outcomes of arterial and venous thromboses and adjusted for the same variables as in the multivariable logistic regression. Stratified results are presented as forest plots. For each stratified analysis, we tested the $\mathrm{P}$ value for equal odds ratios across subgroups by the $\mathrm{P}$ value for an interaction term between sepsis and thrombosis in the model. Regression logistic model showed an interaction between subgroup and the adjusted variables so that the stratified model and interaction model were equivalent. All $\mathrm{P}$ values are two tailed, and confidence intervals 
were calculated at the $95 \%$ level. All statistical analyses were performed with SAS version 9.3 (SAS Institute, Cary, NC).

\section{Results}

In total, 2320920 surgical cases were identified in the ACS-NSQIP cohort during the study period (fig 1). $\Downarrow$ Of these, $3268(0.1 \%)$ were excluded because patients were aged under 18 at time of the surgical procedure. We further excluded 12 $272(0.5 \%)$ cases without available information on the existence of preoperative sepsis. Our final cohort for the analyses comprised 2305380 surgical cases. Preoperative sepsis of any grade was present in 167986 patients (7.3\%): 103479 (4.5\%) had systemic inflammatory response syndrome, 48735 (2.1\%) had sepsis, and 15772 (0.7\%) had severe sepsis/septic shock.

The prevalence of preoperative sepsis was similar across the categories of age, race, and sex (table 1). $\Downarrow$ Preoperative sepsis was more common in patients with diabetes, renal failure, bleeding disorders; smokers; and patients having general surgery. Non-emergency procedures were less common in patients with sepsis.

\section{Risk of postoperative arterial and venous thromboses associated with systemic inflammatory response}

The presence of preoperative sepsis increased the overall risk of postoperative arterial and venous thromboses by three times (odds ratio 3.1, 95\% confidence interval 3.0 to 3.1) (table 2). $\Downarrow$ When we stratified the risk of thrombosis by severity of sepsis, the risk increased with severity: the adjusted odds ratio was 2.5 (95\% confidence interval 2.4 to 2.6 ) in patients with systemic inflammatory response syndrome, 3.3 (3.1 to 3.4) in patients with sepsis, and 5.7 (5.4 to 6.1) in patients with severe sepsis.

\section{Risk of arterial thrombosis}

The odds ratio for arterial thrombosis in patients with any preoperative sepsis was 2.7 (95\% confidence interval 2.5 to 2.8 ) compared with patients without sepsis (table 2 ). $\Downarrow$ The adjusted odds ratios ranged from 2.2 to 5.0 according to the severity of the preoperative sepsis. Although the postoperative risk was similar for both myocardial and stroke (odds ratio $2.7 v 2.6$, respectively), the risk of having a stroke was about two thirds higher than risk of myocardial infarction in patients with preoperative severe sepsis, with odds ratios of 6.1 (5.3 to 6.9) and 4.1 (3.7 to 4.6 ), respectively.

\section{Subgroup analysis for arterial thrombosis}

While none of the baseline characteristics changed the overall association between preoperative sepsis and the risk of postoperative arterial thrombosis, the effect of sepsis seemed to be lower with increasing age (odds ratio 4.5 in patients aged $\leq 40 v 2.0$ in patients $>80$ ) (fig 2). $\Downarrow$ Also, men had a lower risk of arterial thrombosis than women (odds ratio $2.4 v 3.0$, interaction $\mathrm{P}<0.001)$. Similarly, patients with diabetes had a lower risk of arterial thrombosis than those without diabetes. Patients with disseminated cancer and preoperative sepsis had a lower risk of postoperative arterial thrombosis than patients without cancer (odds ratio $1.9 v 2.7, \mathrm{P}=0.004$ ). Finally, patients undergoing cardiac surgery had a lower risk of arterial thrombosis compared with other subspecialties.

We found no effect modification for BMI, smoking status, or type of anaesthesia (general $v$ local). Emergency, non-emergency, elective, and non-elective procedure categories also all had a similar odds ratio between 1.9 and 2.0, without significant effect modification $(\mathrm{P}>0.10)$.

Among patients without diabetes, the proportion of arterial thrombosis increased from $0.40 \%$ in those without preoperative sepsis to $1.34 \%$ in those with preoperative sepsis (odds ratio 3.4). Among patients with diabetes, the proportion increased from $1.07 \%$ to $2.49 \%$, respectively (odds ratio 2.4 ). Among patients without cancer, the proportion of arterial thrombosis increased from $0.49 \%$ in those without preoperative sepsis to $1.56 \%$ in those with preoperative sepsis (odds ratio 3.2). Among patients with cancer, the proportion increased from 0.87 to $1.83 \%$, respectively (odds ratio 2.1 ).

\section{Risk of venous thrombosis}

When we evaluated the risk of postoperative venous thrombosis specifically, the overall risk was higher than for arterial thrombosis, with an odds ratio of 3.3 (95\% confidence interval 3.2 to 3.4) in case of preoperative sepsis (table 2). $\Downarrow$ This risk ranged from 2.6 to 6.1 according to severity. The risk of postoperative deep venous thrombosis was about two thirds higher than the risk of pulmonary embolism when a sepsis was present before the surgical procedure (odds ratio $3.7 \vee 2.4$, respectively). This difference of risk increased with severity of sepsis, with almost a 2.5 times higher risk of deep venous thrombosis than pulmonary embolism in patients with severe sepsis.

\section{Subgroup analysis for venous thrombosis}

The risk of postoperative venous thrombosis associated with sepsis was not affected by most baseline characteristics (fig 3). $\Downarrow$ The risk was constant across and within all subgroups, except for patients with a disseminated cancer or a bleeding disorder and patients from a cardiac subspecialty $(\mathrm{P}<0.001$ for interaction). The risk of venous thrombosis was also independently increased in both emergency and non-emergency procedures, but with a significant higher risk for non-emergency procedures (odds ratio $3.3 v 2.1, \mathrm{P}<0.001$ ). Conversely, for elective procedures in patients with preoperative sepsis there was a twofold increased odds for postoperative venous thrombosis (odds ratio $2.0,95 \%$ confidence interval 1.8 to 2.1 ) in the 2011-12 year dataset $(\mathrm{n}=986034)$. The odds ratio was significantly higher for patients undergoing non-elective procedures (3.3, 2.8 to 3.9 ).

Among patients without cancer, the proportion of venous thrombosis increased from $0.74 \%$ in those without preoperative sepsis to $2.66 \%$ in those with preoperative sepsis (odds ratio 3.7). Among patients with cancer, the proportion increased from $2.77 \%$ to $4.82 \%$, respectively (odds ratio 1.8 ). The proportion of venous thrombosis was therefore higher in patients with cancer $(4.82 \% v 2.66 \%)$, but the association between sepsis and venous thrombosis was less strong (odds ratio $3.7 v 1.8$ ). Among patients with non-emergency procedure, the proportion of venous thrombosis increased from $0.74 \%$ in those without preoperative sepsis to $2.73 \%$ in those with preoperative sepsis (odds ratio 3.8). In comparison, among patients with emergent procedure, the proportion increased from $1.26 \%$ to $2.73 \%$, respectively (odds ratio 2.2).

\section{Secondary analyses}

Compared with patients who did not fulfil any of the criteria for preoperative sepsis, those with preoperative sepsis had a higher risk of 30 day postoperative mortality (adjusted odds ratio $9.2,95 \%$ confidence interval 9.0 to 9.5 ). The odds ratios ranged from 5.9 (5.7 to 6.1) for patients with systemic 
inflammatory response syndrome to 7.6 (7.3 to 7.9$)$ for patients with sepsis and up to 42.3 (40.6 to 44.0) for patients with severe sepsis.

\section{Discussion}

In this large observational cohort of 2.3 million surgical procedures performed across the US from 2005 to 2012, preoperative sepsis was an important independent risk factor for both arterial and venous thromboses, with about three times the risk of each of these complications compared with patients without evidence of preoperative sepsis. Risk was increased more than twofold among those with a systemic inflammation response without proved infection and became higher as the severity of sepsis increased. This increased risk of postoperative thrombosis was present in patients undergoing elective as well as emergency operations and persisted in analyses stratified by age, sex, smoking status, blood pressure, and diabetes. Increased risks for postoperative arterial and venous thromboses were associated with preoperative infection or inflammation among patients with and without cancer and were present in all surgical specialties evaluated.

Our results make clinical sense, as we could expect a potential increased risk of thrombosis in patients with sepsis. To our

knowledge, however, previous research has not determined the burden of arterial or venous thrombosis in the context of sepsis, particularly in surgical patients. Studies have focused on critically ill patients and most were limited in size..$^{15} \mathrm{We}$ quantified the risk for both arterial and venous thromboses and evaluated the contribution from the degree of clinical systemic inflammation.

Although it might at first seem surprising that the risk of venous thrombosis is lower in subgroups of patients with cancer and in cardiac specialties (fig 3), $\Downarrow$ these results can be explained by a typically large use of thromboprophylaxis in such patients because of their underlying risk for thrombosis. Moreover, a higher relative increase risk among patients without cancer and those undergoing non-emergency procedures, but with a lower absolute risk as shown in our results, might also contribute to these findings.

\section{Infection, inflammation, and arterial thrombosis}

While some early case-control studies suggested a link between specific infections and an increased risk of myocardial infarction and stroke, these effects have generally not been observed in larger prospective cohort studies with better adjustment for confounding. Furthermore, several randomised trials of antibiotic treatment failed to show that eradication of infection reduced arterial event rates. ${ }^{16}$ Abundant evidence from the past 20 years, however, has shown that individuals with a pro-inflammatory response are at significantly increased risks for myocardial infarction and stroke, both on a short and long term basis. ${ }^{17-21}$ Acute infection induces such a pro-inflammatory response, and, in our cohort, we found that sepsis was independently associated with an odds ratio of 2.2 to 5.0 for arterial thrombosis. This is comparable with the effect magnitude of most important predictors of perioperative cardiac risk, such as a history of congestive heart failure, a history of cerebrovascular disease, and high risk surgery, that have an odds ratio between 1.9 and 2.8. ${ }^{122}$

In contrast with the latter factors, more action could be taken to reduce cardiovascular risk in patients with sepsis. Strategies to improve outcomes might include improving cardiovascular protection in these patients and, whenever possible, postponement of the surgical procedure as much as possible to treat the sepsis first. While an urgent surgical procedure might be the most appropriate treatment for sepsis, in our study many surgical procedures coded as elective were performed in patients with sepsis. Randomised trials have shown treatment with statins can substantially reduce arterial vascular events among individuals selected for a pro-inflammatory response as detected by increased concentrations of $\mathrm{C}$ reactive protein. ${ }^{23}$

\section{Infection, inflammation, and venous thrombosis}

Evidence to support the use of thromboprophylaxis in patients with sepsis relies mainly on data extrapolated from critically ill patients. ${ }^{24}$ Patients in intensive care units have an increased risk of venous thromboembolism. ${ }^{25-27}$ With a prevalence of sepsis between $17 \%$ and $52 \%$ in these populations, the rationale was that patients with severe sepsis would be at similar or higher risk. While such recommendation exists for general critically ill patients with severe sepsis, no specific recommendation exists specifically for surgical patients with any severity of sepsis. The American College of Chest Physicians (ACCP) recommendations for thromboprophylaxis in surgical patients are based on risk stratification. ${ }^{2}$ The prediction models, however, are not well validated. One of the two recommended prediction models - the Rogers score-doesn't include sepsis or a pro-inflammatory response as predictors. ${ }^{28}$ Sepsis is part of the Caprini model but adds little risk. ${ }^{29}{ }^{30}$ Based on this model, an isolated risk factor of sepsis would attribute a low risk of venous thromboembolism (around $0.7 \%$ ). This contrasts with the $2.7 \%$ prevalence found in our large cohort study (see table $2 \Downarrow$ ). As only half of critically ill patients with sepsis are known to receive thromboprophylaxis, ${ }^{31}$ many surgical patients with sepsis might benefit from thromboprophylaxis as a simple and efficient way of preventing venous thromboembolism. ${ }^{32}$ As with arterial thrombosis, randomised placebo controlled trials have shown that treatment with statins reduces the risk of deep vein thrombosis and pulmonary embolism among individuals selected for a heightened pro-inflammatory response. ${ }^{33}$

\section{Physiopathological assumptions}

Although a causal role is not yet proved, several investigations support the hypothesis that inflammation contributes to atherogenesis. ${ }^{5-34}$ Sepsis could induce a higher risk of arterial and venous thromboses by several possible mechanisms: endothelial injury and dysfunction from endotoxin release; platelet aggregation and a procoagulant state; changes in lipid metabolism; and smooth muscle proliferation. Recent work also implicates leucocyte activation, mast cells, and monocytes in atherogenesis. ${ }^{35}{ }^{36}$ Partly on this basis, two multinational trials are now under way directly testing the inflammatory hypothesis of atherothrombosis: the National Institutes of Health funded cardiovascular inflammation reduction trial (CIRT) testing low dose methotrexate and the industry funded canakinumab anti-inflammatory thrombosis outcomes study (CANTOS) evaluating interleukin- $1 \beta .{ }^{37}$ It seems unlikely that hypotension and hypoxaemia secondary to sepsis play a major role as these factors would be less likely to result in venous thromboembolism. Therefore, other factors that could concomitantly explain both arterial and venous thromboses are more likely.

\section{Limitations of study}

This study has several limitations. Firstly, we used an observational cohort that limits our ability to evaluate 
associations. The dataset, however, is large, multi-institutional, and of high quality. Furthermore, the effect gradient across levels of sepsis severity provides strong support for our hypothesis. Secondly, we were unable to use a mixed effect model to account for hospital clustering, although this would only slightly increase the confidence interval and not modify the effect estimates. Thirdly, we had no information about drugs patients were taking. For example, patients taking statins might be less likely to have an arterial or venous thrombosis, and patients taking anticoagulants and antithrombotic treatments might be less likely to have venous thrombosis. We also did not have data on genetic determinants of thrombosis such as factor $\mathrm{V}$ Leiden, which is known to increase rates of deep vein thrombosis and pulmonary embolism. Fourthly, because of increases in cardiac troponin in some cases of sepsis, we cannot completely exclude an overdiagnosis of arterial thrombosis in these patients, although an isolated increase in troponin was not a sufficient criterion to determine the presence of arterial thrombosis. Finally, despite the presence of an exhaustive list of factors collected in this cohort and the careful selection of the covariates to adjust for, we cannot fully exclude residual confounding.

\section{Implications}

Our results have several clinical implications. Firstly, though it is obvious to avoid any elective surgical procedure in patients with sepsis and severe sepsis, it is interesting to see here that even patients with systemic inflammatory response syndrome have a significantly increased risk of postoperative thrombosis. Therefore, elective procedure should be avoided as much as possible in such patients, and a careful benefit-risk evaluation of the surgical procedure should include any grade of sepsis as a risk factor for both arterial and venous thromboses. Secondly, thromboprophylaxis should be considered in surgical patients with preoperative sepsis. Although sepsis has been found to be a risk factor for venous thrombosis in medical patients, our findings support consideration of thromboprophylaxis in surgical patients with preoperative sepsis. A trial would be helpful to definitively address this issue. Finally, cardiovascular protection should be promoted in patients with preoperative sepsis according to their other risk factors.

\section{Conclusions}

Preoperative sepsis was a major determinant of postoperative arterial and venous thromboses in almost all surgical settings. This risk was already clinically important in early stages of sepsis without a proved infectious agent and increased with the severity of sepsis. The risk of arterial and venous thromboses should be recognised in preoperative patients with any grade of sepsis, and postponement of the surgical procedure should be considered whenever possible. Also, adequate thromboprophylaxis and cardiac protection should be considered in all patients presenting with preoperative sepsis of any severity.

We thank E John Orav for his advice on the statistical analysis regarding an earlier version of this manuscript.

The American College of Surgeons National Surgical Quality Improvement Program and the hospitals participating in the ACS NSQIP are the source of the data used herein; they have not verified and are not responsible for the statistical validity of the data analysis or the conclusions derived by the authors.

Contributors: JD designed the study, collected the data, and drafted the manuscript. All authors were involved in the analysis, interpretation of the data, revision of the manuscript for important intellectual content, and in the final approval of the manuscript. JD is guarantor. All authors had full access to all of the data (including statistical reports and tables) in the study and can take responsibility for the integrity of the data and the accuracy of the data analysis.

Funding: JD was partly supported by the Swiss National Science Foundation for his time. The Swiss Science National Foundation had no role in the design and conduct of this study, the analysis or interpretation of the data, or the preparation of this manuscript. Competing interests: All authors have completed the ICMJE uniform disclosure at www.icmje.org/coi_disclosure.pdf and declare: no support from any organisation for the submitted work; JD does consultancy for Profility and Homeward Health; PR is listed as a co-inventor on patents held by the Brigham and Women's hospital that relate to the use of inflammatory biomarkers in cardiovascular disease and diabetes that have been licensed to Siemens and AstraZeneca, has received investigator initiated research grants from the National Institutes of Health, Amgen, Novartis, Pfizer, and AstraZeneca, and has served as a consultant to Vascular Biogenics, ISIS, Aegerion, Lilly, and BostonHeart. DB is a board member of SAE Medical, does consultancy for Zynx, and has received grants from Earlysense and received royalties from Medicalis.

Ethical approval: The study protocol was approved by the institutional review board of Brigham and Women's Hospital/Partners Healthcare (protocol 2013-P-000654).

Transparency: The lead author (the manuscript's guarantor) affirms that the manuscript is an honest, accurate, and transparent account of the study being reported; that no important aspects of the study have been omitted; and that any discrepancies from the study as planned (and, if relevant, registered) have been explained.

Data sharing: No additional data available.

1 Ashton CM, Petersen NJ, Wray NP, Kiefe Cl, Dunn JK, Wu L, et al. The incidence of perioperative myocardial infarction in men undergoing noncardiac surgery. Ann Intern Med 1993;118:504-10.

2 Gould MK, Garcia DA, Wren SM, Karanicolas PJ, Arcelus JI, Heit JA, et al. Prevention of VTE in nonorthopedic surgical patients: antithrombotic therapy and prevention of thrombosis. 9th ed. American College of Chest Physicians Evidence-Based Clinical Practice Guidelines. Chest 2012;141:e227S-77S.

3 Devereaux PJ, Bradley D, Chan MT, Walsh M, Villar JC, Polanczyk CA, et al. An international prospective cohort study evaluating major vascular complications among patients undergoing noncardiac surgery: the VISION Pilot Study. Open Med 2011;5:e193-200.

4 Ridker PM, Cushman M, Stampfer MJ, Tracy RP, Hennekens $\mathrm{CH}$. Inflammation, aspirin, and the risk of cardiovascular disease in apparently healthy men. $N$ Engl $J$ Med 1997;336:973-9.

5 Libby P, Ridker PM, Hansson GK. Progress and challenges in translating the biology of atherosclerosis. Nature 2011;473:317-25.

6 Ross R. Atherosclerosis-an inflammatory disease. N Engl J Med 1999;340:115-26.

7 Epstein SE, Zhou YF, Zhu J. Infection and atherosclerosis: emerging mechanistic paradigms. Circulation 1999;100:e20-8.

8 Vallance P, Collier J, Bhagat K. Infection, inflammation, and infarction: does acute endothelial dysfunction provide a link? Lancet 1997;349:1391-2.

9 Danesh J, Collins R, Peto R. Chronic infections and coronary heart disease: is there a link? Lancet 1997;350:430-6.

10 Fong IW. Emerging relations between infectious diseases and coronary artery disease and atherosclerosis. CMAJ 2000;163:49-56.

11 American College of Surgeons. ACS NSQIP: Program Overview, 2012. http://site.acsnsqip. org/wp-content/uploads/2012/11/NSQIP-Overview-10.12.pdf.

12 Von Elm E, Altman DG, Egger M, Pocock SJ, Gotzsche PC, Vandenbroucke JP. Strengthening the reporting of observational studies in epidemiology (STROBE) statement: Strengthening the reporting of observational studies in epidemiology (STR
guidelines for reporting observational studies. BMJ 2007;335:806-8.

13 Bone RC, Balk RA, Cerra FB, Dellinger RP, Fein AM, Knaus WA, et al. Definitions for sepsis and organ failure and guidelines for the use of innovative therapies in sepsis. The ACCP/SCCM Consensus Conference Committee. American College of Chest Physicians/Society of Critical Care Medicine. Chest 1992;101:1644-55.

14 Graham JW, Donaldson SI. Evaluating interventions with differential attrition: the importance of nonresponse mechanisms and use of follow-up data. J Appl Psychol 1993;78:119-28.

15 Levine RL, LeClerc JR, Bailey JE, Monberg MJ, Sarwat S. Venous and arterial thromboembolism in severe sepsis. Thromb Haemost 2008;99:892-8.

16 Andraws R, Berger JS, Brown DL. Effects of antibiotic therapy on outcomes of patients with coronary artery disease: a meta-analysis of randomized controlled trials. JAMA 2005;293:2641-7.

17 Paganini-Hill A, Lozano E, Fischberg G, Perez Barreto M, Rajamani K, Ameriso SF, et al. Infection and risk of ischemic stroke: differences among stroke subtypes. Stroke 2003;34:452-7.

18 Saikku P, Leinonen M, Tenkanen L, Linnanmaki E, Ekman MR, Manninen V, et al. Chronic Chlamydia pneumoniae infection as a risk factor for coronary heart disease in the Helsinki Heart Study. Ann Intern Med 1992;116:273-8. 


\section{What is already known on this topic}

Arterial and venous thromboses are common serious postoperative complications

Systemic infections with their consequent inflammation and hypercoagulable state might affect risk of postoperative thrombosis

\section{What this study adds}

Preoperative sepsis was an important independent risk factor for both arterial and venous thromboses, with about three times the risk of each of these complications compared with patients without evidence of preoperative sepsis

Risk was more than twofold among those with a systemic inflammation response without proved infection and became higher as the severity of sepsis increased

This increased risk of postoperative thrombosis was present in patients undergoing elective as well as emergency operations

19 Meier CR, Jick SS, Derby LE, Vasilakis C, Jick H. Acute respiratory-tract infections and risk of first-time acute myocardial infarction. Lancet 1998;351:1467-71.

20 Mattila KJ, Valtonen VV, Nieminen MS, Asikainen S. Role of infection as a risk factor for atherosclerosis, myocardial infarction, and stroke. Clin Infect Dis 1998;26:719-34.

21 Lindsberg PJ, Grau AJ. Inflammation and infections as risk factors for ischemic stroke. Stroke 2003;34:2518-32.

22 Lee TH, Marcantonio ER, Mangione CM, Thomas EJ, Polanczyk CA, Cook EF, et al. Derivation and prospective validation of a simple index for prediction of cardiac risk of major noncardiac surgery. Circulation 1999;100:1043-9.

23 Ridker PM, Danielson E, Fonseca FA, Genest J, Gotto AM Jr, Kastelein JJ, et al. Rosuvastatin to prevent vascular events in men and women with elevated C-reactive protein. N Engl J Med 2008;359:2195-207.

24 Dellinger RP, Levy MM, Rhodes A, Annane D, Gerlach H, Opal SM, et al. Surviving sepsis campaign: international guidelines for management of severe sepsis and septic shock: 2012. Crit Care Med 2013;41:580-637.

25 Cade JF. High risk of the critically ill for venous thromboembolism. Crit Care Med 1982;10:448-50.

26 Pingleton SK, Bone RC, Pingleton WW, Ruth WE. Prevention of pulmonary emboli in a respiratory intensive care unit: efficacy of low-dose heparin. Chest 1981;79:647-50.

27 Hirsch DR, Ingenito EP, Goldhaber SZ. Prevalence of deep venous thrombosis among patients in medical intensive care. JAMA 1995;274:335-7.

28 Rogers SO Jr, Kilaru RK, Hosokawa P, Henderson WG, Zinner MJ, Khuri SF. Multivariable predictors of postoperative venous thromboembolic events after general and vascular surgery: results from the patient safety in surgery study. J Am Coll Surg 2007;204:1211-21.

29 Caprini JA, Arcelus JI, Hasty JH, Tamhane AC, Fabrega F. Clinical assessment of venous thromboembolic risk in surgical patients. Semin Thromb Hemost 1991;17 Suppl 3:304-12.

30 Caprini JA. Thrombosis risk assessment as a guide to quality patient care. Dis Mon 2005;51:70-8.
31 Shorr AF, Williams MD. Venous thromboembolism in critically ill patients. Observations from a randomized trial in sepsis. Thromb Haemost 2009;101:139-44.

32 Gardlund B. Randomised, controlled trial of low-dose heparin for prevention of fata pulmonary embolism in patients with infectious diseases. The Heparin Prophylaxis Study Group. Lancet 1996;347:1357-61.

33 Glynn RJ, Danielson E, Fonseca FA, Genest J, Gotto AM, Jr., Kastelein JJ, et al. A randomized trial of rosuvastatin in the prevention of venous thromboembolism. $N$ Engl J Med 2009;360:1851-61.

34 Hansson GK. Inflammation, atherosclerosis, and coronary artery disease. N Engl J Med 2005;352:1685-95

35 Swirski FK, Nahrendorf M. Leukocyte behavior in atherosclerosis, myocardial infarction, and heart failure. Science 2013;339:161-6.

36 Ghattas A, Griffiths HR, Devitt A, Lip GY, Shantsila E. Monocytes in coronary artery disease and atherosclerosis: where are we now? J Am Coll Cardiol 2013:62:1541-51.

37 Ridker PM. Targeting inflammatory pathways for the treatment of cardiovascular disease. Eur Heart $J 2013$.

Accepted: 18 August 2014

\section{Cite this as: BMJ 2014;349:g5334}

This is an Open Access article distributed in accordance with the Creative Commons Attribution Non Commercial (CC BY-NC 3.0) license, which permits others to distribute, remix, adapt, build upon this work non-commercially, and license their derivative works on different terms, provided the original work is properly cited and the use is non-commercial. See: http://creativecommons.org/licenses/by-nc/3.0/. 


\section{Tables}

Table 1/ Characteristics of cohorts of surgical cases $(n=2305380)$ according to presence of sepsis before surgery. Figures are numbers (percentage) unless stated otherwise

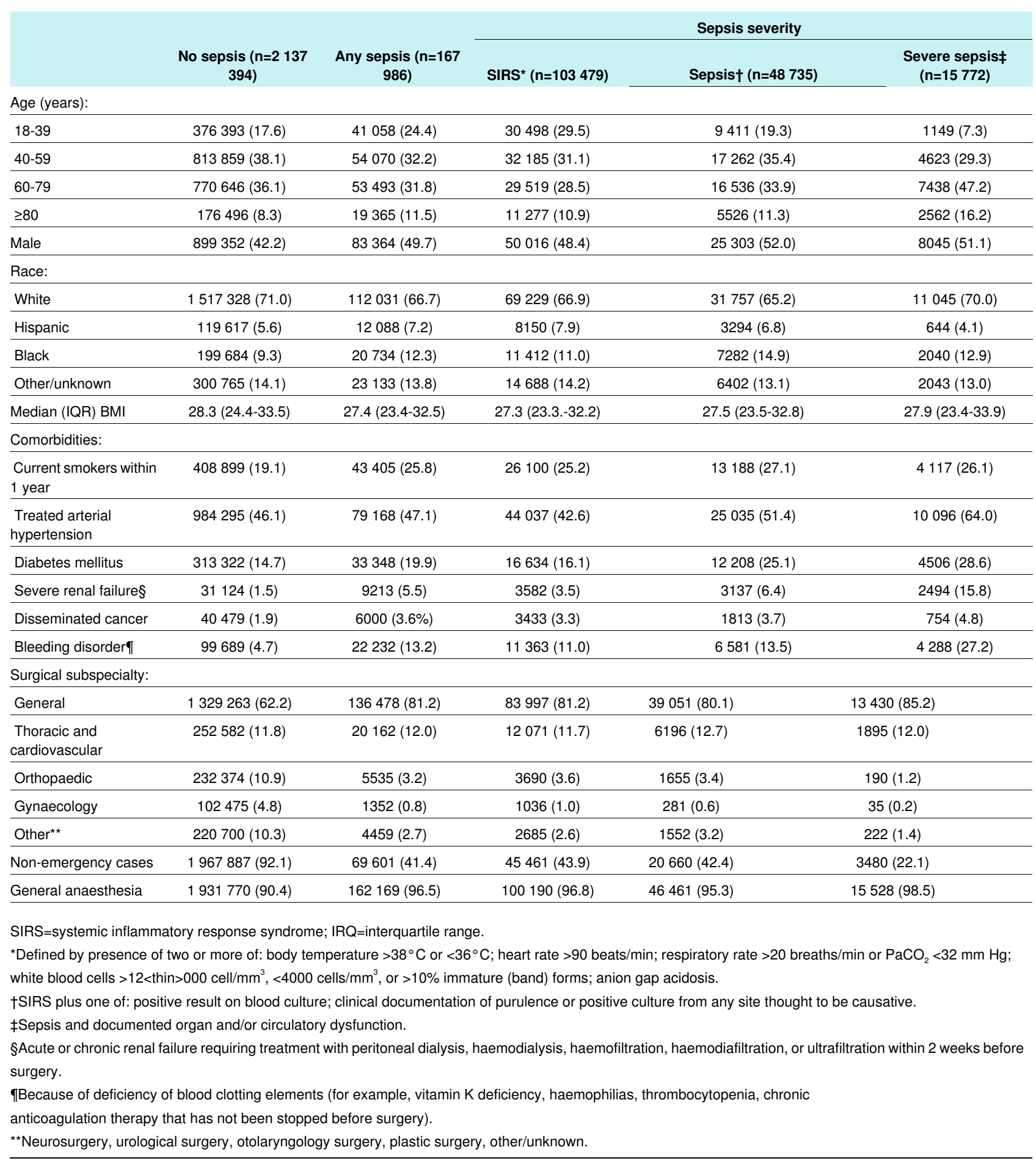


Table 2/ Crude and adjusted odds ratios (95\% confidence interval) for having thrombosis within $\mathbf{3 0}$ days after intervention according to presence of sepsis before surgery

Sepsis severity

Any sepsis $(n=167986)$

$\operatorname{SIRS}^{*}(n=103479) \quad$ Sepsist $(n=48735)$

Severe sepsis $¥(n=15772)$

Composite endpoint for arterial or venous thromboses

\begin{tabular}{|c|c|c|c|c|}
\hline No (\%) & $7018(4.2)$ & $3272(3.2)$ & $2220(4.6)$ & $1526(9.7)$ \\
\hline Crude odds ratio & 3.5 (3.4 to 3.5$)$ & 2.6 (2.5 to 2.7 ) & 3.8 (3.6 to 4.0$)$ & 8.5 (8.0 to 9.0$)$ \\
\hline Adjusted odds ratioß & 3.1 (3.0 to 3.1$)$ & 2.5 (2.4 to 2.6$)$ & 3.3 (3.1 to 3.4$)$ & 5.7 (5.4 to 6.1$)$ \\
\hline \multicolumn{5}{|l|}{ Arterial thrombosis } \\
\hline No (\%) & $2632(1.6)$ & $1247(1.2)$ & $771(1.6)$ & $614(3.9)$ \\
\hline Crude odds ratio & 3.2 (3.0 to 3.3 ) & 2.4 (2.3 to 2.6$)$ & 3.2 (3.0 to 3.5$)$ & 8.1 (7.5 to 8.8$)$ \\
\hline Adjusted odds ratioß & 2.7 (2.5 to 2.8$)$ & 2.2 (2.1 to 2.4 ) & 2.6 (2.4 to 2.8 ) & $5.0(4.6$ to 5.4$)$ \\
\hline \multicolumn{5}{|l|}{ Myocardial infarction } \\
\hline No (\%) & $1643(1.0)$ & $746(0.7)$ & $545(1.1)$ & $352(2.2)$ \\
\hline Crude odds ratio & 3.3 (3.1 to 3.5$)$ & 2.4 (2.2 to 2.6$)$ & 3.8 (3.5 to 4.1$)$ & $7.6(6.8$ to 8.5$)$ \\
\hline Adjusted odds ratioß & 2.6 (2.5 to 2.8$)$ & 2.1 (2.0 to 2.3$)$ & 2.9 (2.6 to 3.1$)$ & 4.1 (3.7 to 4.6$)$ \\
\hline \multicolumn{5}{|l|}{ Stroke } \\
\hline No (\%) & $1056(0.6)$ & $536(0.5)$ & $244(0.5)$ & $276(1.8)$ \\
\hline Crude odds ratio & 3.0 (2.8 to 3.3$)$ & 2.5 (2.3 to 2.7$)$ & 2.4 (2.1 to 2.8$)$ & $8.6(7.6$ to 9.7$)$ \\
\hline Adjusted odds ratioß & 2.7 (2.5 to 2.9$)$ & 2.4 (2.2 to 2.6$)$ & 2.1 (1.9 to 2.4$)$ & 6.1 (5.3 to 6.9$)$ \\
\hline \multicolumn{5}{|l|}{ Venous thrombosis } \\
\hline No (\%) & $4590(2.7)$ & $2121(2.1)$ & $1509(3.1)$ & $960(6.1)$ \\
\hline Crude odds ratio & 3.6 (3.5 to 3.7$)$ & 2.7 (2.6 to 2.8$)$ & 4.1 (3.9 to 4.3 ) & $8.3(7.7$ to 8.8$)$ \\
\hline Adjusted odds ratio§ & 3.3 (3.2 to 3.4$)$ & 2.6 (2.5 to 2.7$)$ & 3.7 (3.5 to 3.9$)$ & 6.1 (5.7 to 6.5$)$ \\
\hline \multicolumn{5}{|l|}{ Pulmonary embolism } \\
\hline No (\%) & $1208(0.7)$ & $657(0.6)$ & $377(0.8)$ & $174(1.1)$ \\
\hline Crude odds ratio & 2.4 (2.2 to 2.6$)$ & 2.1 (2.0 to 2.3 ) & 2.6 (2.4 to 2.9$)$ & $3.8(3.2$ to 4.4$)$ \\
\hline Adjusted odds ratioß & 2.4 (2.3 to 2.6$)$ & 2.2 (2.0 to 2.4$)$ & 2.6 (2.3 to 2.9$)$ & 3.1 (2.6 to 3.6$)$ \\
\hline \multicolumn{5}{|c|}{ Deep venous thrombosis } \\
\hline No (\%) & $3719(2.2)$ & $1641(1.6)$ & $1237(2.5)$ & $841(5.3)$ \\
\hline Crude odds ratio & 4.1 (3.9 to 4.2$)$ & 2.9 (2.8 to 3.1$)$ & 4.7 (4.4 to 5.0$)$ & $10.2(9.5$ to 11.0$)$ \\
\hline Adjusted odds ratioß & 3.7 (3.5 to 3.8$)$ & 2.8 (2.7 to 3.0$)$ & 4.2 (3.9 to 4.4$)$ & $7.2(6.7$ to 7.8$)$ \\
\hline
\end{tabular}

SIRS=systemic inflammatory response syndrome.

*Defined by presence of two or more of: body temperature $>38^{\circ} \mathrm{C}$ or $<36^{\circ} \mathrm{C}$; heart rate $>90$ beats/min; respiratory rate $>20 \mathrm{breaths} / \mathrm{min}$ or PaCO $<32 \mathrm{~mm} \mathrm{Hg}$; white blood cells $>12<$ thin $>000$ cell $/ \mathrm{mm}^{3},<4000$ cells $/ \mathrm{mm}^{3}$, or $>10 \%$ immature (band) forms; anion gap acidosis.

†SIRS plus one of: positive result on blood culture; clinical documentation of purulence or positive culture from any site thought to be causative.

‡Sepsis and documented organ and/or circulatory dysfunction.

§Adjusted for age, sex, diabetes, smoking status, treated arterial hypertension, renal failure, cancer, bleeding disorder or anticoagulation, general anesthesia,

surgical subspecialty. 


\section{Figures}

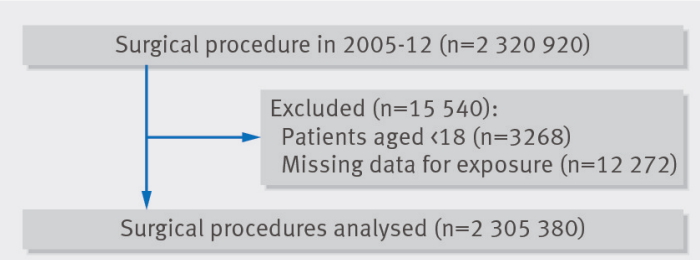

Fig 1 Flow of participants in study of impact of sepsis on risk of postoperative arterial and venous thromboses diagram

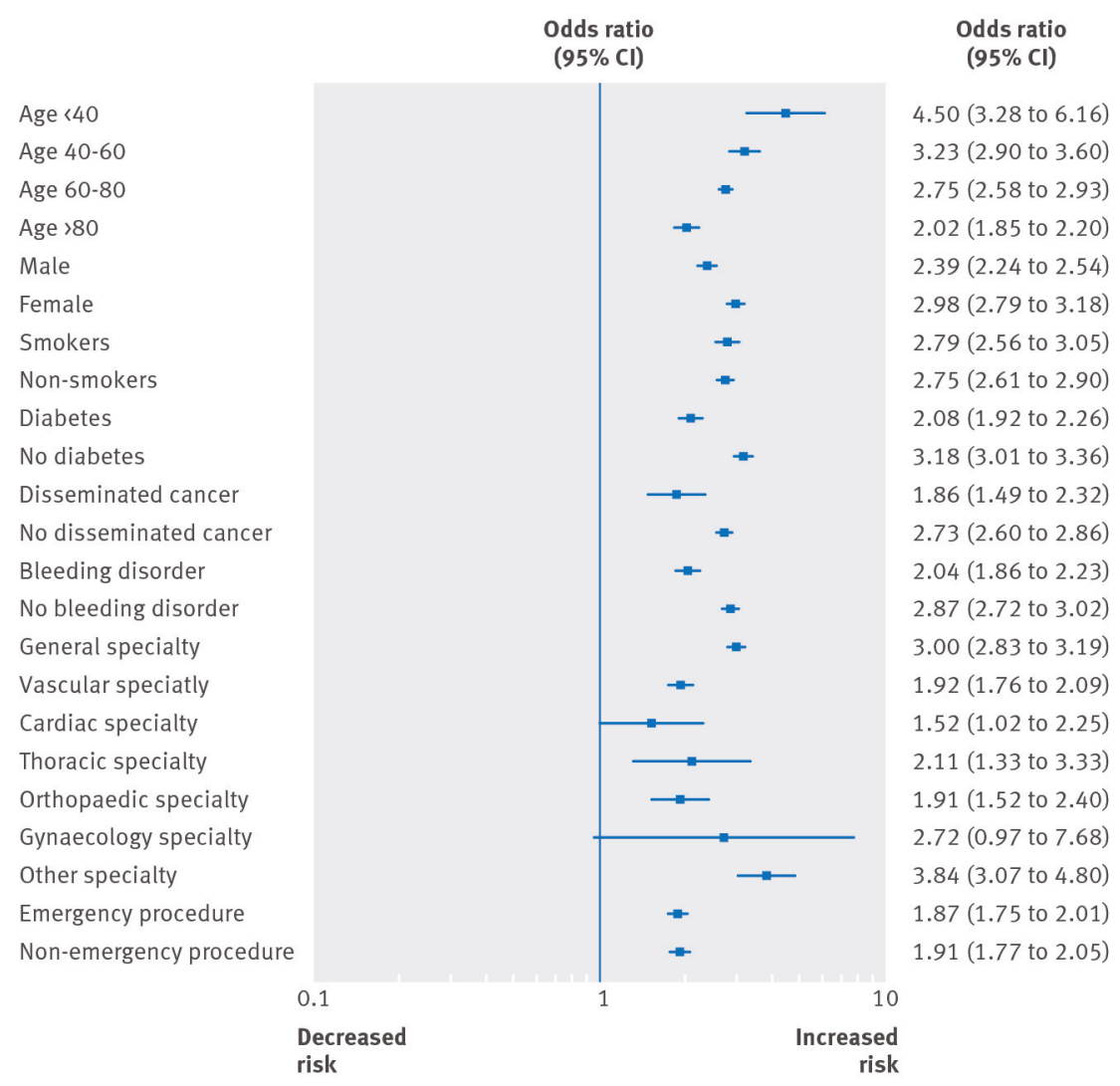

Fig 2 Subgroup analysis for arterial thrombosis risk associated with preoperative sepsis. Arterial thrombosis includes acute myocardial infarction or stroke that occurred intraoperatively or within 30 days after surgical procedure. Odds ratios adjusted for age, sex, diabetes, smoking status, treated arterial hypertension, renal failure, cancer, bleeding disorder or anticoagulation, general anaesthesia, and surgical subspecialty 


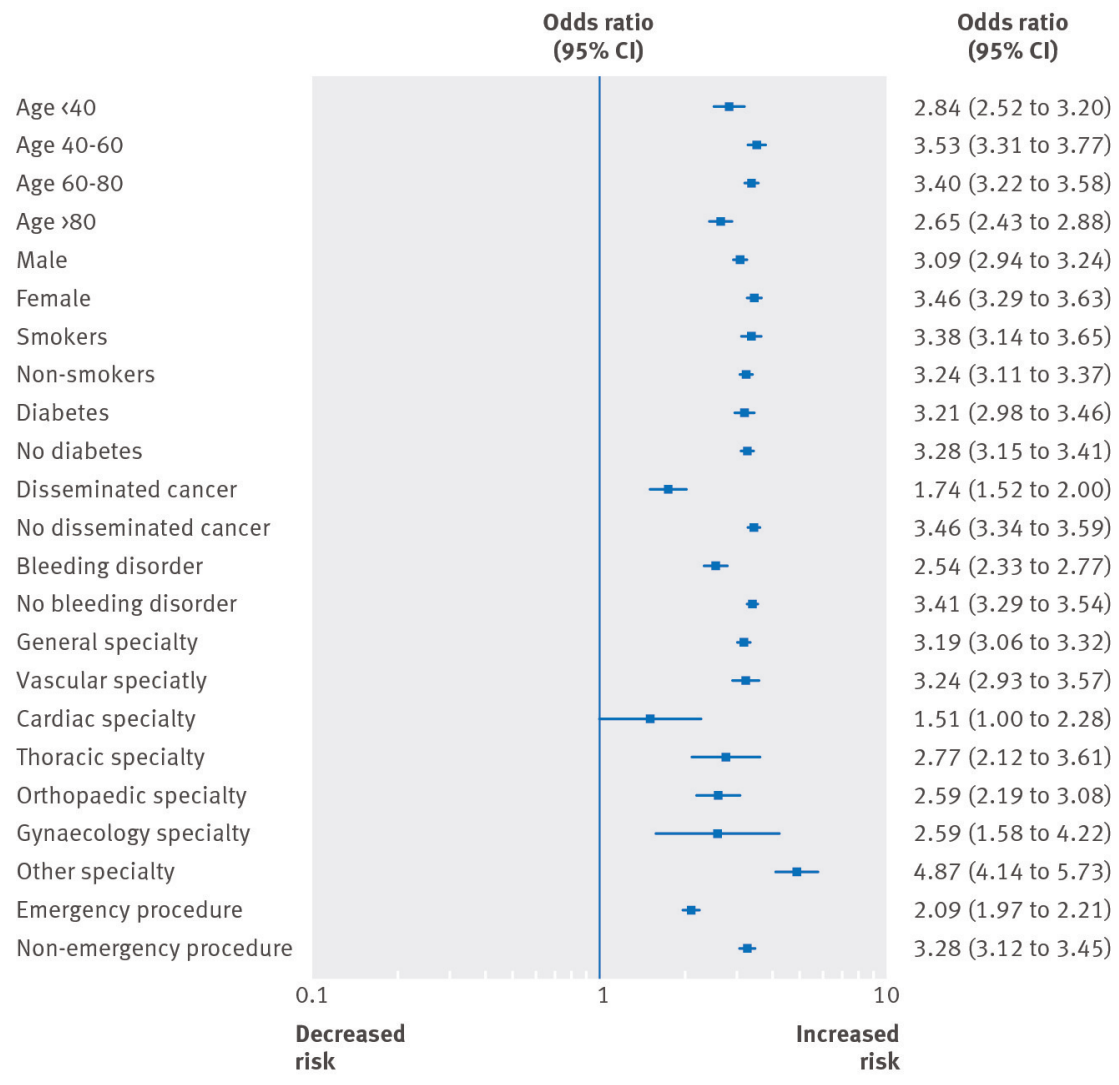

Fig 3 Subgroup analysis for risk of venous thrombosis associated with preoperative sepsis. Venous thrombosis includes deep venous thrombosis or pulmonary embolism that occurred intraoperatively or within 30 days after surgical procedure. Odds ratios adjusted for age, sex, diabetes, smoking status, treated arterial hypertension, renal failure, cancer, bleeding disorder or anticoagulation, general anaesthesia, and surgical subspecialty 